\title{
STUDIES OF PROPERTIES OF RFID TAGS USED IN MECHATRONIC AND LOGISTIC SYSTEMS
}

\author{
P. Zajac*, W. Skoczynski**, W. Romanowicz***
}

\begin{abstract}
The subject of this paper is research task consisting in determining the characteristics of RFID systems in the conditions of variable factors that can be found in the real-world operating conditions of such systems logistic, mechatronic systems Kwasniowski, Zajac, Zajac (2011) - with each of them having its own manufacturer's product sheet. After a brief review of the literature, a research station for typical RFID tags was designed based on the Arduino board; software for communication of both systems was developed using typical software libraries. Subsequently, these systems were activated. A valuable part of the paper is research on the characteristics of the RFID system. Original is the way of conducting the tests, the means of measurement used and the results of tests. The obtained results are interesting, especially the conclusion that the working area declared by the manufacturer is not consistent with the obtained measurement results. The information contained in the summary of the paper may be helpful/useful in planning the use of RFID tags in real-world conditions.
\end{abstract}

Keywords: RFID, automatic identification systems, logistics systems

\section{Introduction}

Passive RFID tags used in logistics systems or mechatronic systems use the energy of the reader's magnetic field for correct operation (as already described in paper Zajac, Kowalczyk (2017)). The basic read-write processes depend on the following conditions: position of the tag relative to the reader. If the transponder coil is exposed to an alternating magnetic field of intensity $\mathrm{H}$, which induces a voltage $\mathrm{u}_{0}$ in the coil when the main axes of the coils are parallel to each other, the relationship described in equation (1) occurs when the angle between the axes changes by $\vartheta$.

$$
u_{o \vartheta}=u_{o} \cdot \cos (\vartheta)
$$

Figure 1a shows the coils of the reader and transponder, their main axes and the angle between them. This relationship affects the reading area, which decreases with angle change, as in fig. $1 \mathrm{~b}$.

System sensitivity (environment). RFID uses wireless connections; the sensitivity depends on the operating environment. These include: impact of materials present in the system area on electromagnetic waves, such as diffraction and interference of waves; in addition, there are phenomena of multiple reflections, deflection and attenuation of waves. In addition, factors affecting the distribution of electromagnetic fields include the geometry and construction of the rooms in which the system operates, influence of water and metals on wave propagation (in some cases the operating range is over three times smaller than the free space range). Atmospheric conditions. RFID systems are resistant to atmospheric conditions such as: temperature, pollution, humidity or light intensity. Collisions between tags. The reader receives overlapping waves, which prevent correct reading of the data carried by them. In order to

\footnotetext{
Dr Eng. Pawel Zajac: Faculty of Mechanical Engineering, Wrocław University of Technology; 27 Wybrzeze Wyspianskiego st.; PL, pawel.zajac@pwr.edu.pl

** Dr Eng. Waclaw Skoczynski: Faculty of Mechanical Engineering, Wrocław University of Technology; 27 Wybrzeze Wyspianskiego st.; 50-370, Wrocław; PL

*** Eng. Wojciech Romanowicz: Faculty of Mechanical Engineering, Wrocław University of Technology; 27 Wybrzeze Wyspianskiego st.; 50-370, Wrocław; PL
} 
minimize collisions in RFID systems, anti-collision algorithms are introduced, e.g. Aloha algorithm. Quantities of transponders in the reading area. Every transponder in the antenna range consumes energy. The amount of energy emitted by the reader is limited, so the more tags in the vicinity of the reader, the smaller the range of operation of the reader. Movements (simultaneous or not) of the reader and transponder relative to each other. This issue was widely described in the papers Zajac, Kwasniowski (2017). An important factor here is speed; excessive speed of the tag or reader will cause that the presence of the tag in the reader area will be too short for the reader to read the information from the tag; this is referred to as "tag loss".

a)

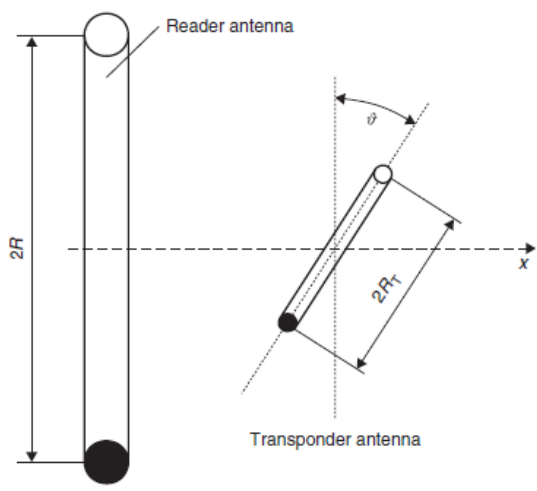

b)

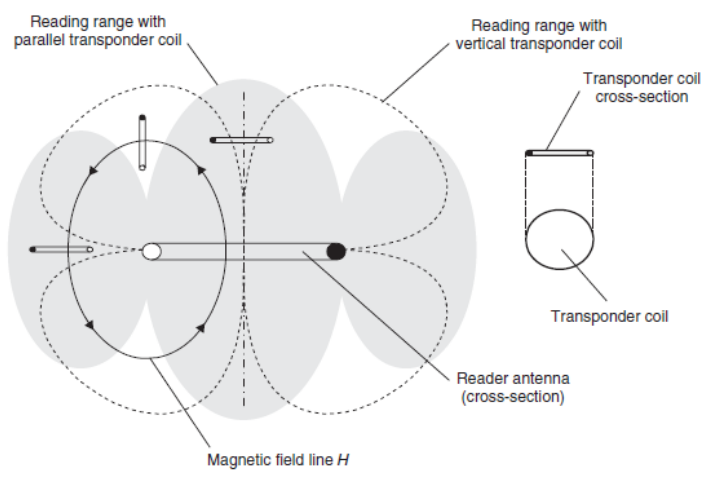

Fig. 1: a) Cross-section of the coils of the reader and the transponder with a marked angle between them influencing the induced voltage in the transponder Zajac (2015); b) Operation area depending on the coils orientation.

\section{Measuring station concept}

The base of the RFID test station will be Arduino Leonardo, which acts as a reader - i.e. ATmega32u4 microcontroller with 20 input/output pins, of which 12 are analog inputs and 7 can be used to generate a PWM (Pulse-Width Modulation) signal. The clock frequency of the microprocessor is $16 \mathrm{MHz}$. The Arduino board is also equipped with microUSB, power input and microcontroller reset button, being therefore a complete prototype board for fast construction of electronic circuits. The Arduino's programming language is standardized and based on the widely used $\mathrm{C}$ language. Arduino IDE is used for programming. Passive transponders operate at low and medium frequency, i.e. $125 \mathrm{KHz}-13.56 \mathrm{MHz}$. The most popular in logistics systems is the MiFare standard. For compatibility with Arduino, the system is powered by $5 \mathrm{~V}$ DC voltage. Ready-made manufacturer's libraries have been used in the test station, which eliminates possible programming errors. For the purpose of the tests, the PN532 RFID module was selected, which is powered by voltage from $3.3 \div 5 \mathrm{~V}$ and communicates via I2C, SPI or UART.

a)

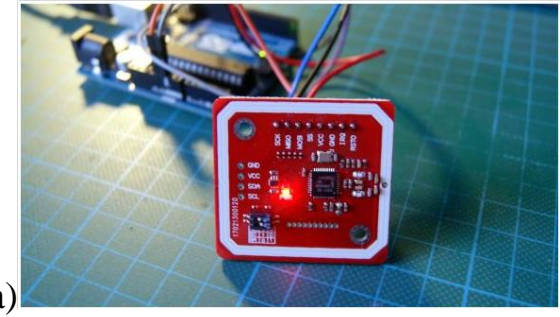

b)

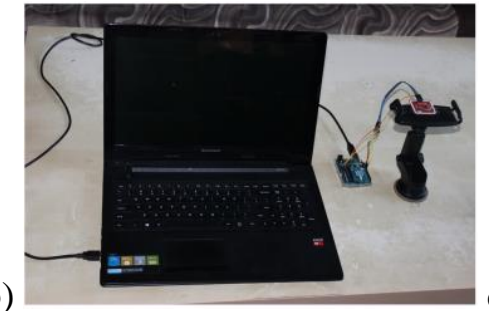

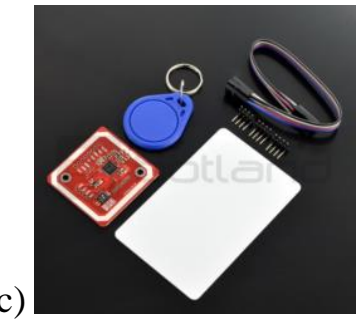

Fig. 2: a) PN532 module connected to Arduino; b) Measuring station consisting of a computer, Arduino and PN532 module; c) RFID PN532 module together with a card and key ring performing the function of tags.

Due to the frequency of MiFare standard $(13.56 \mathrm{MHz})$, the module has its own oscillator with a frequency of $27.12 \mathrm{MHz}$. The technical specification is included in the product sheet. For MiFare standard, the communication speed of the reader with the transponder can reach up to $424 \mathrm{kbit} / \mathrm{s}$. Manufacturerspecified range is max. $5 \mathrm{~cm}$. The set also includes two tags in the shape of a card and a key ring. The whole is shown in Fig. 1c. SPI bus was used for communication between the PN532 module and Arduino. Then, the module was connected to Arduino in accordance with www.arduino.cc. 
Fig. 2a shows the PN532 module configured in SPI operating mode. To minimize the impact of wires connecting the reader to Arduino, the reader was set at an angle of $90^{\circ}$ to the main axis of the antenna, which is hidden under the white paint shown in fig. $2 b$.

\section{Analysis of results. Conclusions}

Before the measurements were carried out, the planning of the experiment was performed in accordance with experiment planning theory. Due to volume constraints of the paper, this issue was omitted. In Table 1 the results together with the measurement uncertainties for 3 different transponders are collected.

Tab. 1: Average values including uncertainty of measurement of max. range, reading time and max. angle.

\begin{tabular}{|c|c|c|r|r|r|c|}
\hline Identifier & \multicolumn{2}{|c|}{ Card 1 } & \multicolumn{2}{c|}{ Card 2 } & \multicolumn{2}{c|}{ Key ring } \\
\hline Measurement & Distance $[\mathrm{mm}]$ & Angle $\left[{ }^{\circ}\right]$ & Distance $[\mathrm{mm}]$ & Angle $\left[{ }^{\circ}\right]$ & Distance $[\mathrm{mm}]$ & Angle $\left[{ }^{\circ}\right]$ \\
\hline Basic test & $21,38 \pm 2.19$ & $36,7 \pm 5,36$ & $18,95 \pm 1,80$ & $39,4 \pm 3,47$ & $12,77 \pm 1,23$ & $30,6 \pm 2,50$ \\
\hline Cardboard & $19,11 \pm 1.20$ & $25,4 \pm 4,40$ & $17,45 \pm 1,11$ & $29,8 \pm 3,55$ & $11,49 \pm 1,56$ & $21,4 \pm 2,67$ \\
\hline Plastic & $20,58 \pm 1.21$ & $32,9 \pm 3,73$ & $19,31 \pm 0,92$ & $34,5 \pm 3,10$ & $13,75 \pm 0,67$ & $30,1 \pm 2,60$ \\
\hline Cotton & $19,74 \pm 0.90$ & $32,8 \pm 3,71$ & $18,65 \pm 0,88$ & $32,6 \pm 2,63$ & $13,55 \pm 1,04$ & $26,2 \pm 2,39$ \\
\hline Water & $20,66 \pm 1.78$ & $31,2 \pm 2,9$ & $19,73 \pm 1,22$ & $30,4 \pm 2,01$ & $13,43 \pm 1,10$ & $25,0 \pm 2,21$ \\
\hline Spinach & $19,6 \pm 1.06$ & $30,3 \pm 2,63$ & $18,58 \pm 1,02$ & $30,2 \pm 1,62$ & $13,45 \pm 1,19$ & $24,2 \pm 2,57$ \\
\hline
\end{tabular}

The maximum distance for correct reading of the identifier in the basic test for the new MiFare card was $21.38 \pm 22.19 \mathrm{~mm}$, and is smaller than the range declared by the manufacturer, which is to be up to $50 \mathrm{~mm}$. As evident from the chart for card 2, which was used on a daily basis, the range is smaller and amounts to $18.95 \pm 1.80 \mathrm{~mm}$. The difference in the maximum range between the two cards is $2.43 \mathrm{~mm}$, so it is significant. Therefore, it can be concluded that the range is affected by wear and tear of the elements of the identifier. Probably the most important role will be played by a capacitor, on which the voltage induced in the coil is stored. When the capacitor is worn out, the voltage stored in the capacitor decreases. According to the manufacturer, the maximum voltage generated in the transponder is $3.6 \mathrm{~V}$ and the minimum necessary for proper operation is $1.6 \mathrm{~V}$. Comparing a brand new card (card 1) with the identifier in the shape of a key ring, a large difference in the operating range, amounting to as much as $8.61 \mathrm{~mm}$, can be observed. This leads to a conclusion than the range of the key ring is $40 \%$ smaller than the range of the card, which is dependent on the magnetic field strength and distance from the coil versus coil radius.

In the extended test, the influence of selected materials between the reader and transponder on the reading performance was studied fig. 3 - shows that most of the materials used in the measurements did not have a significant impact on the maximum range of the RFID system. An evident difference was noted, however, in the maximum distance between a brand new card and a used one, as well as the key ring in not only the basic measurements described above, but also in the extended test, which clearly shows that the wear and tear and the size of the antenna have an impact on the range and thus on the operation of the RFID system.

The materials selected for tests were materials that occur in the real-world environment of RFID systems, but also those that may interfere with the operation of the system or impair its properties such as reading range or reading efficiency measured as reading time. Hence, the first choice was cardboard, which is ubiquitous in every industry. According to the preliminary findings cardboard should not affect the behavior of the RFID system, as it is made of cellulose; however, as shown in fig. 3a, 3c and 3d, the maximum distance of correct reading as well as the maximum angle is smaller than the value from the basic test. After analyzing the literature related to the production of cardboard, including studies carried out in Zajac (2015), it can be concluded that the coating can be covered with a printing ink containing metal admixtures, and the cardboard used in the food industry may contain a thin layer of aluminum (as confirmed by the known reflection phenomenon) radio waves).

In the extended test, the influence of water on the reading of the RFID system was examined. It was assumed that, as in the case of microwaves which are perfectly absorbed by water, as well as in the case of waves of the frequency used in the study, the absorption of waves by water will be visible in the results. However, there are no deviations from the values obtained in the basic test, neither for the cards nor for the key ring. Materials such as plastic, cotton and organic matter, in our case spinach, also do not influence the operation and range of the RFID system. 

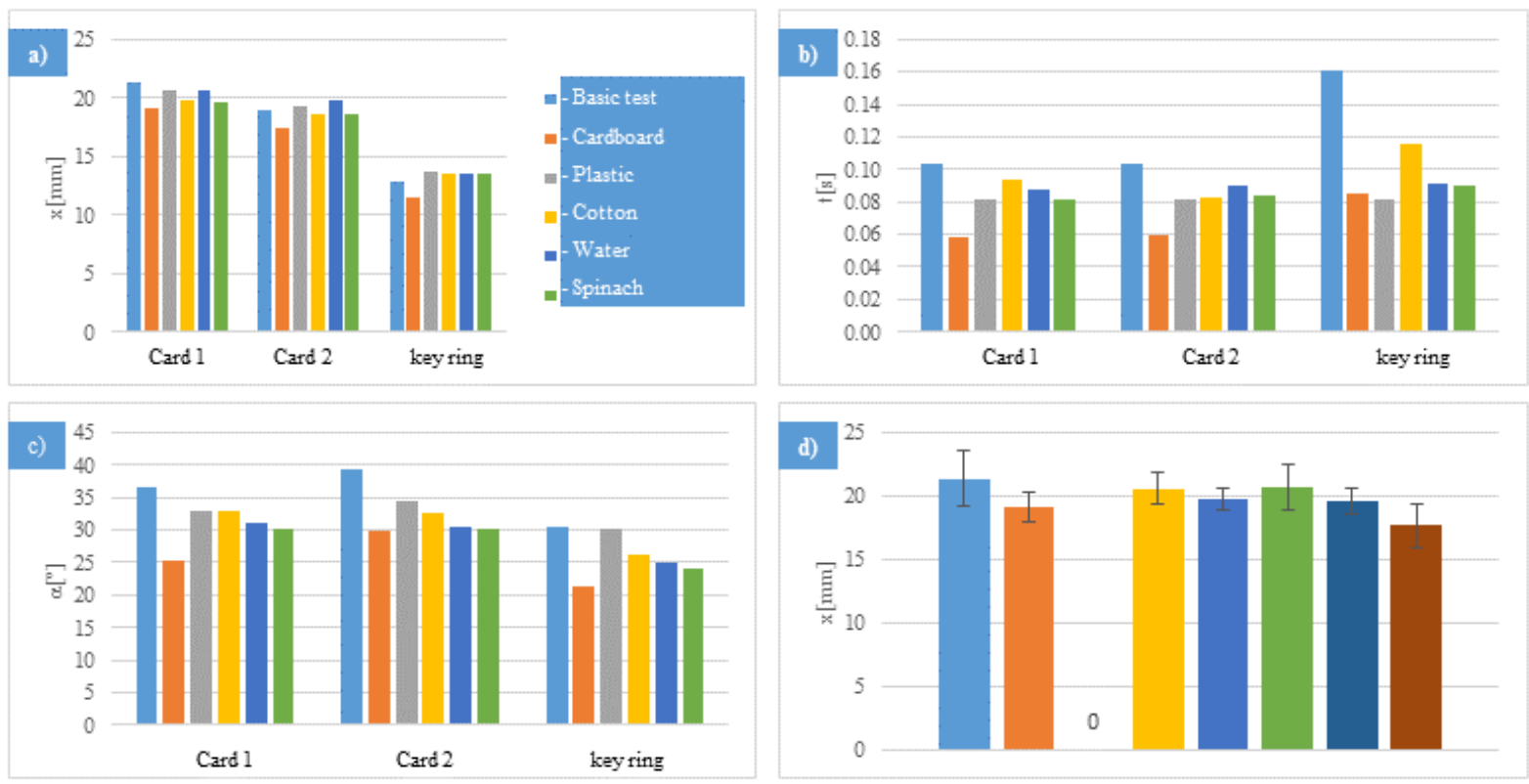

Fig. 3: a)Maximal RFID ranges for different materials within the range of the RFID system; b) Reading times for three different identifiers with different materials; c) Maximum measured angle at which the correct reading of information written on the tag for different materials and different identifiers was possible; d) Maximum average measured distance at which the correct reading of the identifier took place with marked measurement uncertainties for card 1.

Figure 3d shows the extended test results for a brand new MiFare card. As mentioned above, only cardboard had a statistically significant impact on the range of RFID system. In the graph, the measurement of the maximum distance at more than one identifier present in the working area was also included. As is evident from the figure, the presence of two transponders reduces the working area of the RFID system. This is due to the limitation of energy consumed and emitted by the reader. As the number of transponders in the working area increases, the amount of energy absorbed by the transponders increases, while the energy emitted by the reader is constant. The energy absorbed by the identifier weakens the magnetic field strength and, as mentioned above, there is a certain limit to the minimum magnetic field strength that is necessary for the correct operation of the transponder. Considering the above, it can be assumed that with the increase in the number of transponders in the vicinity of the reader, the range of the reader will be smaller. Another aspect affecting the reliability of the RFID system associated with multiple tags is the reading time. For many identifiers, reading time increases with the number of tags in the workspace. Because the system does not send a request to a specific identifier, it only sends a general signal to which all transponders in the reader's range respond. Additionally, the extension of this time is influenced by the collision of tags. This occurs when several tags respond to the reader's signal at the same time and their responses overlap.

\section{References}

Kwasniowski, S., Zajac, M., Zajac, P. (2011) Telematic problems of unmanned vehicles positioning at container terminals and warehouses. In: 10th conference, TST 2010 (Mikulski, J. ed). Berlin, Springer, pp. 391-399.

Zajac, P., Kowalczyk, L. (2017) Comprehensive service of conference participants using automatic identification. In: Engineering Mechanics 2017:23rd international conference (Vladimír, F. ed). Brno, pp. 1090-1093.

Zajac, P., Kwasniowski, S. (2017) The reliability problems of reading RFID tags in logistics warehouse systems. In: 21st Innovative Manufacturing Engineering \& Energy International Conference, IManE\&E 2017 (Slătineanu, L. eds.), EDP Sciences, pp. 1-6, art. 05012, (MATEC Web of Conferences, ISSN 2261-236X; vol. 112)

Zajac, P. (2015) Evaluation of automatic identification systems according to ISO $50001: 2011$. In: Progress in automation, robotics and measuring techniques: control and automation (Szewczyk, R., Zielinski, C., Kaliczynska, M. eds.). Springer, pp. 345-355. (Advances in Intelligent Systems and Computing, ISSN 21945357; vol. 350). 